\title{
Semiconductor Nanowires and Nanotubes: From Fundamentals to Diverse Applications
}

\author{
Qihua Xiong, ${ }^{1}$ Craig A. Grimes, ${ }^{2}$ Margit Zacharias, ${ }^{3}$ Anna Fontcuberta i Morral, ${ }^{4}$ \\ Kenji Hiruma, ${ }^{5}$ and Guozhen Shen ${ }^{6}$ \\ ${ }^{1}$ School of Physical and Mathematical Sciences, Nanyang Technological University, 21 Nanyang Link, Singapore 637371 \\ ${ }^{2}$ State Key Laboratory of Materials-Oriented Chemical Engineering, Nanjing University of Technology, Nanjing, \\ Jiangsu 210009, China \\ ${ }^{3}$ Faculty of Applied Science (IMTEK), University of Freiburg, Georges-Köhler-Allee 103, 79110 Freiburg, Germany \\ ${ }^{4}$ Ecole Polytechnique Federale de Lausanne (EPFL), 1015 Lausanne, Switzerland \\ ${ }^{5}$ Research Center for Integrated Quantum Electronics, Hokkaido University, North 13, West 8, Sapporo 060-8628, Japan \\ ${ }^{6}$ Wuhan National Laboratory for Optoelectronics, Huazhong University of Science and Technology, 1037 Luoyu Road, \\ Wuhan 430074, China
}

Correspondence should be addressed to Qihua Xiong, qihua@ntu.edu.sg

Received 24 October 2011; Accepted 24 October 2011

Copyright ( 2012 Qihua Xiong et al. This is an open access article distributed under the Creative Commons Attribution License, which permits unrestricted use, distribution, and reproduction in any medium, provided the original work is properly cited.

Research in the field of semiconductor nanowires (SNWs) and nanotubes has been progressing into a mature subject with several highly interdisciplinary subareas such as nanoelectronics, nanophotonics, nanocomposites, biosensing, optoelectronics, and solar cells. SNWs represent a unique system with novel properties associated to their one-dimensional (1D) structures. The fundamental physics concerning the formation of discrete 1D subbands, coulomb blockade effects, ballistic transport, and many-body phenomena in 1D nanowires and nanotubes provide a strong platform to explore the various scientific aspects in these nanostructures. A rich variety of preparation methods have already been developed for generating well-controlled 1D nanostructures and from a broad range of materials. The present special issue focuses on the recent development in the mechanistic understanding of the synthesis, the studies on electrical/optical properties of nanowires and their applications in nanoelectronics, nanophotonics, and solarenergy harvesting.

In this special issue, we have several invited review articles and contributed papers that are addressing current status of the fundamental issues related to synthesis and the diverse applications of semiconducting nanowires and nanotubes. One of the papers reviews the progress of the top-down approach of developing silicon-based vertically aligned nanowires to explore novel device architectures and integration schemes for nanoelectronics and clean energy applications. Another paper reviews the recent developments and experimental evidences of probing the confined optical and acoustic phonon in nonpolar semiconducting (Si and $\mathrm{Ge}$ ) nanowires using Raman spectroscopy. The paper by K. Hiruma et al. spotlights the III-V semiconductor nanowires and demonstrates selective-area metal organic vapor phase epitaxy grown $\mathrm{GaAs} / \mathrm{In}(\mathrm{Al}) \mathrm{GaAs}$ and $\mathrm{InP} / \mathrm{InAs} / \mathrm{InP}$ nanowires with heterojunctions along their axial and radial directions. The paper written by K. Tateno et al. also reviews the vapor-liquid-solid (VLS-) grown coremultishell $\mathrm{Ga}(\mathrm{In}) \mathrm{P} / \mathrm{GaAs} / \mathrm{GaP}$ nanowires on $\mathrm{Si}$ substrates and introduces an interesting phenomenon of formation of flat-top nanowires achieved by using small-sized Au particles in one growth procedure.

The remaining papers illustrate the research on metal oxides semiconducting materials which are well known for wide band gap and transparent conducting properties. The paper by N. Bao et al. reviews the transparent films of selfassembled $\mathrm{TiO}_{2}$ nanotube arrays, bulk heterojunction solar cells, ordered heterojunction solar cells, and liquid-junction dye-sensitized solar cells. The paper by J. Pan et al. provides a helpful review of the research activities focused on synthesis of $1 \mathrm{D} \mathrm{SnO}_{2}$ nanostructures and their devices applications in gas sensing, lithium-ion batteries, and nanophotonics. The paper by D. Gedamu et al. demonstrates the studies 
of different routes of fabrication of $\mathrm{ZnO}$ semiconducting nanowires between microstructural contacts formed directly on a silicon chip. The paper by C.-T. Liu et al. focuses on the synthesis of $\mathrm{ZnO}$-nanoparticles to fabricate a mask-free thinfilm transistor by ink-jet printing. The emerging process for fabricating printable transistors with $\mathrm{ZnO}$ nanoparticles as the active channel and Poly(4-vinylphenol) matrix as the gate dielectric, respectively, suggests that the printable materials and the printing technology enable the use of all printed lowcost flexible displays for transparent electronic applications.

\author{
Qihua Xiong \\ Craig A. Grimes \\ Margit Zacharias \\ Anna Fontcuberta i Morral \\ Kenji Hiruma \\ Guozhen Shen
}



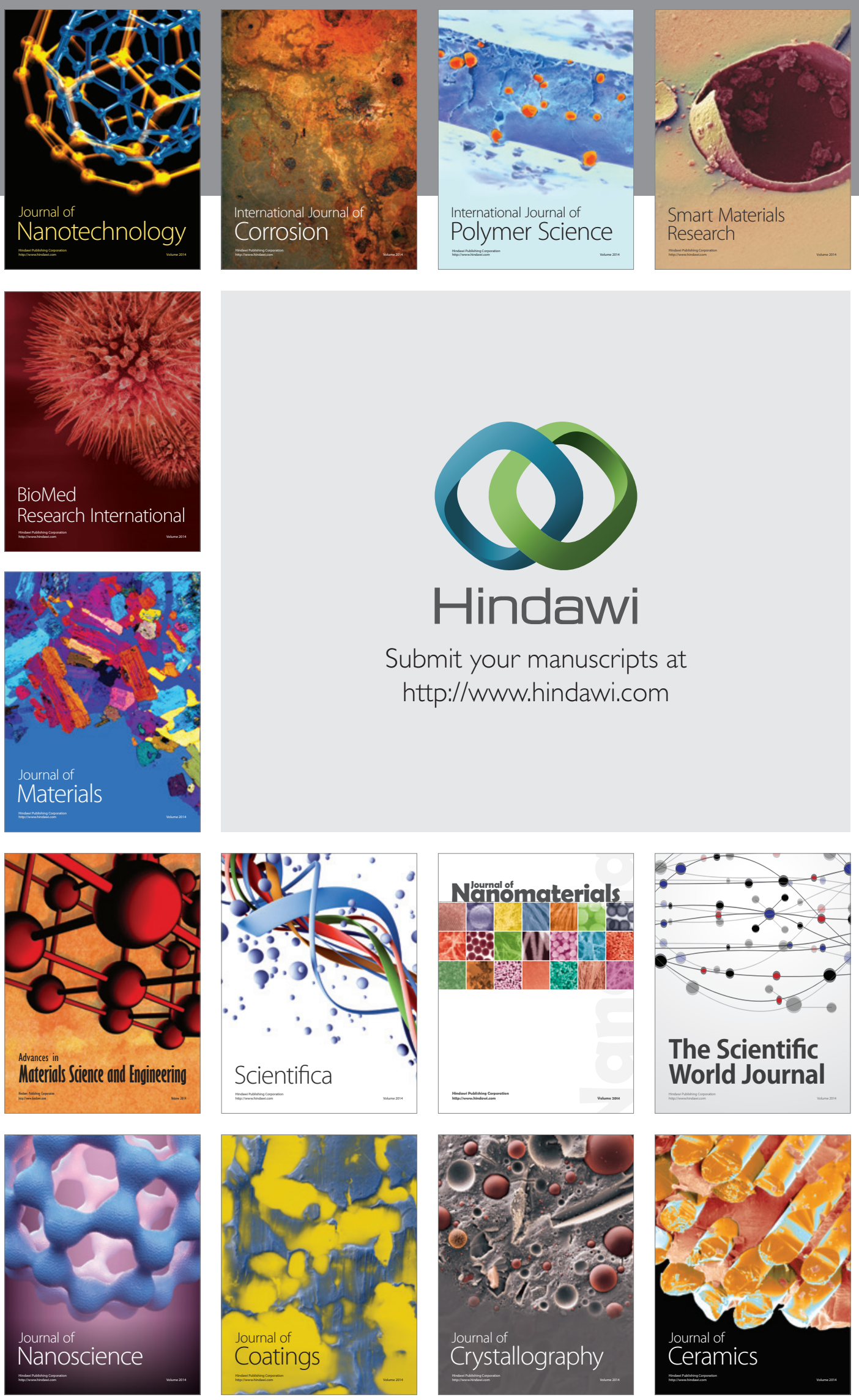

The Scientific World Journal

Submit your manuscripts at

http://www.hindawi.com

\section{World Journal}

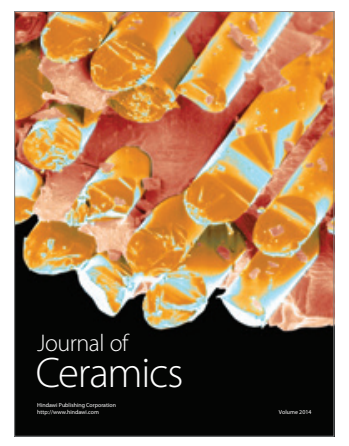

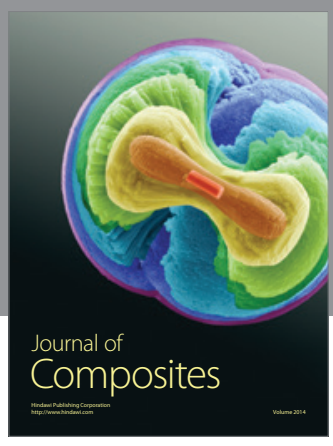
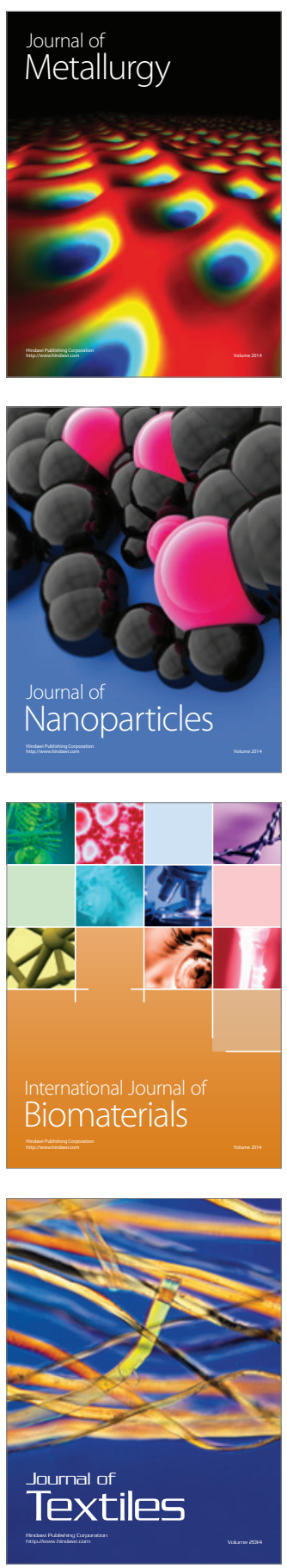